\title{
ESTADO DE HIDRATAÇÃO DE ATLETAS EM CORRIDA DE RUA DE 15 KM SOB ELEVADO ESTRESSE TÉRMICO
}

\author{
HYDRATION STATUS IN ATHLETES ON 15 KM STREET RACE UNDER ELEVATED HEAT STRESS
}

ESTADO DE HIDRATACIÓN DE ATLETAS EN CARRERA DE CALLE DE 15 KM BAJO ELEVADO ESTRÉS TÉRMICO

Ednei Costa Maia' (Médico)

Herikson Araújo Costa ${ }^{1}$

(Educador Físico)

Jurema Gonçalves Lopes de Castro Filha' (Educador Físico)

Mario Norberto Sevilio de Oliveira Junior' (Educador Físico)

1. Universidade Federal do Maranhão (UFMA), São Luís, MA, Brasil.

\section{Correspondência:}

Rua Engenheiro Brito Passos, 14, Quadra B, Conj Elka, Monte Castelo, São Luís, MA, Brasil. 65032-520. ednei.maia@hotmail.com

\section{RESUMO}

Introdução: Um adequado estado de hidratação é de suma importância para os participantes de corridas de rua, tanto para um melhor desempenho esportivo, quanto na prevenção dos distúrbios causados pelo calor. Objetivo: O estudo visa avaliar o estado de hidratação de indivíduos do sexo masculino em corrida de rua de

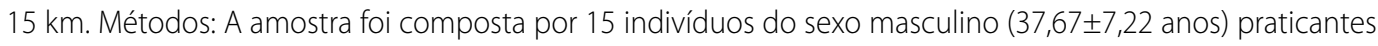
habituais de corrida de rua. $O$ estado de hidratação foi avaliado antes e após a corrida através dos seguintes parâmetros: peso corporal, coloração e gravidade específica da urina (GEU), análise da impedância bioelétrica (BIA), níveis de hemoglobina $(\mathrm{Hb})$ e hematócrito $(\mathrm{Ht})$. Na análise dos dados, foi utilizado o teste $t$ de Student pareado ou o teste de Wilcoxon, aceitando-se como estatisticamente significante $p<0,01$. Resultados: Houve diminuição do peso corporal dos indivíduos após a corrida $(p<0,0001)$, assim como elevação dos níveis de $\mathrm{Hb}$ e Ht. Apesar da diminuição observada na GEU após o exercício, não houve melhora significativa do padrão de coloração da urina ou diferença entre o conteúdo inicial e final de água corporal total. A temperatura ambiente (TA) e umidade relativa do ar (URA) foram de $38,75 \pm 1,79^{\circ} \mathrm{C}$ e $37,37 \pm 4,66 \%$, respectivamente. Conclusão: $O$ exercício no ambiente em questão conduziu os indivíduos a um estado de desidratação, apenas detectado pela análise da variação do peso corporal e de parâmetros sanguíneos através dos níveis de $\mathrm{Hb}$ e Ht. Parâmetros urinários como a coloração da urina e GEU, assim como aqueles obtidos através da BIA, provavelmente foram confundidos por variáveis não controladas pelo presente estudo.

Palavras-chave: exercício, transtornos de estresse por calor, desidratação.

\section{ABSTRACT}

Introduction: An adequate hydration status is of utmost importance to participants of street running, both for better sports performance and to preventi disorders caused by heat. Objective: The study aimed to evaluate the hydration status of males on a $15 \mathrm{~km}$ street race. Methods: The sample consisted of 15 males ( $37.67 \pm 7.22$ years old) habitual street runners. The hydration status was evaluated before and after the race by the following parameters: body weight, color and urine specific gravity (USG), bioelectrical impedance analysis (BIA), hemoglobin (Hb) and hematocrit (Ht). In data analysis, the paired Student t-test or the Wilcoxon test were used, setting as statistically significant $p<0.01$. Results: There was a decrease in body weight of the subjects after the race ( $p<0.0001)$, as well as an increase in $\mathrm{Hb}$ and $\mathrm{Ht}$. Despite the observed decrease in USG after exercise, there was no significant improvement in the standard of urine color or difference between the initial and final contents of total body water. The room temperature ( $R T)$ and relative humidity $(R H)$ were $38.75 \pm 1.79^{\circ} \mathrm{C}$ and $37.37 \pm 4.66 \%$, respectively. Conclusion: The exercise in the environment at issue led the individuals to a state of dehydration, only detected by the analysis of variation in body weight and blood parameters such as $\mathrm{Hb}$ and $\mathrm{Ht}$. Urinary parameters such as urine color and USG, as well as those obtained by BIA, were probably confused by variables that were not controlled by the present study.

Keywords: exercise, heat stress disorders, dehydration.

\section{RESUMEN}

Introducción: Un adecuado estado de hidratación es de suma importancia para los participantes de las carreras de calle, tanto para un mejor desempeño deportivo, como en la prevención de los disturbios causados por el calor. Objetivo: El estudio pretende evaluar el estado de hidratación de individuos del sexo masculino en carrera de calle de $15 \mathrm{~km}$. Métodos: La muestra fue compuesta por 15 individuos masculinos (37,67 \pm 7,22 años) practicantes habituales de carreras de calle. El estado de hidratación fue evaluado antes y después de la carrera a través de los siguientes parámetros: peso corporal, coloración y gravedad especifica de la orina (GEO), análisis de la impedancia bioeléctrica (BIA), niveles de hemoglobina $(\mathrm{Hb})$ y hematocrito $(\mathrm{Ht})$. En el análisis de los datos, fue utilizado el test $t$ de Student emparejado o el test de Wilcoxon, aceptándose como estadísticamente significativo $p<0,01$. Resultados: Hubo disminución del peso corporal de los individuos después de la carrera ( $p<0,0001)$, así como aumento de los niveles de Hb y Ht. A pesar de la disminución observada en la GEO después del ejercicio, no hubo una mejora significativa del patrón de coloración de la orina o diferencia entre el contenido inicial y final de agua corporal total. La temperatura ambiente (TA) y la humedad relativa del aire (HRA) fueron 
de $38,75 \pm 1,79$ y $37,37^{\circ} \mathrm{C} \pm 4,66 \%$, respectivamente. Conclusión: El ejercicio en el ambiente en cuestión llevó a los individuos a un estado de deshidratación, solamente detectado mediante el análisis de la variación del peso corporal y de parámetros sanguíneos como los niveles de Hb y Ht. Parámetros urinarios como coloración de la orina y GEO, así como aquellos obtenidos mediante BIA, probablemente fueron confundidos por variables no controladas en este estudio.

Palabras clave: ejercicio, trastornos de estrés por calor, deshidratación.

\section{INTRODUÇÃO}

Ao praticar exercícios físicos, as pessoas submetem-se a diversas condições ambientais de temperatura, umidade, exposição ao sol, vento, etc. Dependendo da taxa metabólica, condições ambientais e roupas usadas, o exercício pode induzir uma elevação significativa na temperatura corporal (núcleo e pele). Esta elevação produz respostas direcionadas à perda de calor corporal, como maior fluxo sanguíneo cutâneo e secreção aumentada de suor ${ }^{1}$.

Aproximadamente $25 \%$ da energia química proveniente da oxidação de substratos energéticos durante a caminhada rápida ou a corrida se transformam em energia mecânica, aquela responsável pelo movimento, sendo o restante transformado imediatamente em energia térmica. Contudo, inclusive a energia mecânica acaba também sendo transformada em energia térmica posteriormente. Portanto, 100\% da energia são convertidos em calor. A energia térmica que se acumula durante o exercício deve ser dissipada, do contrário o organismo entraria em colapso com superaquecimento corporal em questão de poucos minutos de atividade contínua².

A transferência de calor corporal ao meio externo pode ocorrer através de quatro mecanismos termorregulatórios básicos: radiação, condução, convecção e evaporação. Contudo a evaporação representa a principal defesa contra o superaquecimento, sendo responsável por aproximadamente $80 \%$ da perda total de calor quando o organismo está fisicamente ativo ${ }^{3}$. Assim, a evaporação do suor compreende o mecanismo central de perda de calor durante o exercício vigoroso em clima quente, portanto as perdas através do suor podem ser substanciais.

Durante exercícios de alta intensidade e longa duração, o esforço pode induzir o acometimento dos atletas por doenças provocadas pelo calor, tais como câimbras musculares associadas ao exercício, exaustão pelo calor e colapso pelo calor, o que pode resultar no abandono da atividade ou ainda colapso durante ou após o exercício. A desidratação também ocorre durante o exercício prolongado, sendo mais rápida em ambiente quente, no qual os indivíduos perdem consideravelmente mais suor do que pode ser reposto através da ingestão de líquidos ${ }^{4}$.

O estresse inerente ao exercício é acentuado pela desidratação, que por sua vez associa-se a aumento da temperatura corporal, prejuízo às respostas fisiológicas e ao desempenho físico, bem como a riscos para a saúde 5 . Portanto, o conhecimento do estado de hidratação do indivíduo antes, durante e após o exercício torna-se importante para a prática regular, sendo fundamental para evitar os problemas de saúde decorrentes da desidratação ${ }^{6}$.

Dessa forma, um adequado estado de hidratação é de suma importância para os participantes de corridas de rua, tanto para um melhor desempenho esportivo, quanto na prevenção dos distúrbios causados pelo calor.

O objetivo do presente trabalho foi avaliar o estado de hidratação de indivíduos do sexo masculino em corrida de rua de $15 \mathrm{~km}$.

\section{MÉTODOS}

O estudo teve o projeto aprovado pelo Comitê de Ética em Pesquisa da Universidade Federal do Maranhão (UFMA), Brasil, sob o número de parecer 545.550 (CAAE: 20913014.5.0000.5087), de acordo com as normas da Resolução 196/96 do Conselho Nacional de Saúde. Os objetivos e a metodologia empregada foram previamente informados aos participantes, sendo as dúvidas esclarecidas oportunamente. Todos os participantes assinaram Termo de Consentimento Livre e Esclarecido.

A amostra foi obtida por conveniência e a participação dos indivíduos deu-se de forma voluntária. Inicialmente 26 indivíduos adultos do sexo masculino compareceram ao local de prova. Contudo, um dos indivíduos apresentou níveis de pressão arterial acima de 160×105 mmHg antes da prova e teve sua participação vetada, conforme recomendações da Sociedade Brasileira de Cardiologia ${ }^{7}$. Portanto, 25 indivíduos iniciaram efetivamente a corrida. No entanto, apenas 15 indivíduos concluíram o percurso proposto.

A corrida teve início aproximadamente às $11 \mathrm{~h}$ da manhã e foi realizada nas dependências do Campus Universitário do Bacanga (UFMA), com distância total de 15 km (cinco voltas em percurso de três quilômetros). O consumo hídrico dos participantes ocorreu "ad libitum" através de garrafas com água $(500 \mathrm{~mL})$ de uso individual. O conteúdo restante em cada garrafa ao final da prova foi mensurado para cálculo do volume total de água ingerido. A temperatura ambiente (TA) e umidade relativa do ar (URA) foram monitoradas a cada cinco minutos através de termohigrômetro digital (Modelo HT-260, Instrutherm, São Paulo, Brasil).

O protocolo de pesquisa envolvia em primeiro momento: (1) amostragem de urina em recipientes coletores descartáveis não estéreis; (2) aferição da frequência cardíaca (FC), pressão arterial sistólica (PAS) e diastólica (PAD) de repouso pelo método auscultatório em esfigmomanômetro de coluna de mercúrio (Unitec, São Paulo, Brasil); (3) aferição da estatura e peso corporal em balança eletrônica adulto (W300, Welmy ${ }^{\circledR}$, São Paulo, Brasil) com precisão de 0,5 cm para estatura e 0,05 Kg para o peso; (4) avaliação da composição corporal através de aparelho de bioimpedância tetrapolar (BIA450, Biodynamics ${ }^{\circledR}$, Washington, EUA); (5) amostragem de sangue venoso periférico $(4 \mathrm{~mL})$ em tubos coletores de sangue à vácuo contendo EDTA (Vacuette ${ }^{\circledR}$, Kremsmünster, Austria).

Os referidos procedimentos foram realizados antes do início da corrida e repetidos conforme os indivíduos concluíam a prova, com exceção da aferição da FC, PAS, PAD e estatura que foram realizadas uma única vez para fins de caracterização da amostra. As amostras de urina foram classificadas quanto à coloração por avaliador único através de tabela de coloração da urina ${ }^{8}$. Em seguida, cerca de $50 \mu \mathrm{L}$ de cada amostra de urina foram analisados em refratômetro digital (Modelo RTP-20ATC, Instrutherm, São Paulo, Brasil) para determinação da gravidade específica da urina (GEU), considerando-se os pontos de corte estabelecidos por Casa et al. ${ }^{8}$ (tabela 1). As amostras de sangue foram avaliadas através de analisador diagnóstico automático (ADVIA ${ }^{\circledR} 2120$, Siemens, Tarrytown, EUA).

Os valores foram apresentados como média $(\bar{X})$ e desvio-padrão (DP). As variáveis foram testadas quanto à normalidade através do teste de Shapiro-Wilk. Na comparação dos parâmetros entre os momentos antes e após a corrida, foi utilizado o teste $t$ de Student pareado ou o teste de Wilcoxon. Os dados foram analisados através do software BioEstat versão 5.3, aceitando-se como estatisticamente significante $p<0,01$. 
Tabela 1. Índices de estado de hidratação.

\begin{tabular}{c|c|c|c}
\hline Estado de hidratação & $\begin{array}{c}\text { Variação do peso } \\
\text { corporal (\%) }\end{array}$ & $\begin{array}{c}\text { Coloração } \\
\text { da urina }\end{array}$ & GEU \\
\hline Bem hidratado & +1 a -1 & 1 ou 2 & $<1010$ \\
\hline Desidratação mínima & -1 a -3 & 3 ou 4 & $1010-1020$ \\
\hline Desidratação significativa & -3 a -5 & 5 ou 6 & $1021-1030$ \\
\hline Desidratação grave & $>-5$ & $>6$ & $>1030$ \\
\hline
\end{tabular}

\section{RESULTADOS}

A amostra foi composta por 15 indivíduos adultos do sexo masculino (37,67 \pm 7,22 anos) praticantes habituais de corrida de rua. A tabela 2 apresenta as medidas antropométricas e hemodinâmicas utilizadas na caracterização do grupo avaliado. O tempo médio de prova foi $1 \mathrm{~h} 16 \mathrm{~min} 16 \mathrm{~s} \pm 12 \mathrm{~min} 43 \mathrm{~s}$.

A tabela 3 apresenta a comparação entre os parâmetros de composição corporal obtidos por BIA antes e depois da corrida. Observou-se diminuição significativa do peso corporal dos indivíduos em relação ao início da prova $(\Delta \%=-3,43 \pm 0,84 \%)$, caracterizando desidratação significativa (>-3\% do peso corporal inicial) $)^{8}$.

A ingestão de líquidos nas duas horas que antecederam o início da corrida foi bastante heterogênea entre os indivíduos ( $\mathrm{X} \pm \mathrm{DP}=240 \pm 200,29$ $\mathrm{mL}$ ), sendo o mesmo comportamento observado para o consumo hídrico ao longo da prova ( $\bar{X} \pm D P=493,53 \pm 237,77 \mathrm{~mL}$ ). O volume médio de ingestão hídrica por hora de exercício físico foi de $388,61 \mathrm{~mL} / \mathrm{h}$. Os parâmetros sanguíneos e urinários utilizados na avaliação do estado de hidratação dos indivíduos são apresentados abaixo (tabela 4). A TA registrada no decorrer da prova foi de $38,75 \pm 1,79^{\circ} \mathrm{C}$, enquanto a URA foi de $37,37 \pm 4,66 \%$ (figura 1).

Tabela 2. Idade, medidas antropométricas e hemodinâmicas do grupo avaliado ( $\mathrm{n}=15)$.

\begin{tabular}{c|c}
\hline Variáveis & $\overline{\mathbf{X}} \pm \mathrm{DP}$ \\
\hline Idade (anos) & $37,67 \pm 7,22$ \\
\hline Peso inicial $(\mathrm{kg})$ & $64,20 \pm 7,38$ \\
\hline Estatura $(\mathrm{cm})$ & $168,13 \pm 7,07$ \\
\hline IMC $\left(\mathrm{kg} / \mathrm{m}^{2}\right)$ & $22,67 \pm 1,79$ \\
\hline FCR $(\mathrm{bpm})$ & $55,60 \pm 9,28$ \\
\hline PAS $(\mathrm{mmHg})$ & $122,80 \pm 10,39$ \\
\hline PAD $(\mathrm{mmHg})$ & $80,67 \pm 5,94$ \\
\hline
\end{tabular}

$\overline{\mathrm{X}}$ : média; DP: desvio-padrão; IMC: índice de massa corporal; FCR: frequência cardíaca de repouso; PAS: pressão arterial sistólica; PAD: pressão arterial diastólica.

Tabela 3. Composição corporal dos indivíduos obtida por análise da impedância bioelétrica (BIA).

\begin{tabular}{c|c|c|c|c}
\hline Parâmetros & Antes $(\overline{\mathrm{X}} \pm \mathrm{DP})$ & Depois $(\overline{\mathrm{X}} \pm \mathrm{DP})$ & $\boldsymbol{\Delta}$ & $\mathbf{P}$ \\
\hline Peso $(\mathrm{Kg})$ & $64,20 \pm 7,38$ & $61,99 \pm 7,12$ & $-2,21$ & $<0,0001^{*}$ \\
\hline $\mathrm{MM}(\mathrm{Kg})$ & $53,31 \pm 6,61$ & $53,27 \pm 6,41$ & $-0,04$ & $0,3635^{* *}$ \\
\hline $\mathrm{MM}(\%)$ & $83,1 \pm 4,58$ & $86,04 \pm 5,33$ & 2,94 & $<0,01^{* *}$ \\
\hline $\mathrm{MG}(\mathrm{Kg})$ & $10,86 \pm 3,02$ & $8,71 \pm 3,36$ & $-2,15$ & $<0,01^{*}$ \\
\hline $\mathrm{MG}(\%)$ & $16,9 \pm 4,58$ & $13,96 \pm 5,33$ & $-2,94$ & $<0,01^{* *}$ \\
\hline $\mathrm{Al}(\mathrm{L})$ & $24,45 \pm 3,10$ & $25,12 \pm 3,34$ & 0,67 & $0,2014^{*}$ \\
\hline $\mathrm{AE}(\mathrm{L})$ & $13,94 \pm 2,13$ & $13,43 \pm 2,42$ & $-0,51$ & $0,0468^{* *}$ \\
\hline $\mathrm{ACT}(\mathrm{L})$ & $38,39 \pm 4,73$ & $38,55 \pm 4,62$ & 0,16 & $0,8647^{* *}$ \\
\hline $\mathrm{IMC}\left(\mathrm{kg} / \mathrm{m}^{2}\right)$ & $22,67 \pm 1,79$ & $21,89 \pm 1,73$ & $-0,78$ & $<0,0001^{*}$ \\
\hline
\end{tabular}

$\overline{\mathrm{X}}$ : média; DP: desvio-padrão; $\Delta$ : variação; : probabilidade de significância; MM: massa magra; MG: massa gorda; Al: água intracelular; AE: água extracelular; ACT: água corporal total; IMC: índice de massa corporal; ( $\left.{ }^{*}\right)$ : teste $t$ de Student pareado; $\left(^{*}\right)$ : teste de Wilcoxon.

Tabela 4. Comparação de variáveis urinárias e sanguíneas antes e após a corrida.

\begin{tabular}{c|c|c|c}
\hline Parâmetros & Antes $(\overline{\mathrm{X}} \pm \mathrm{DP})$ & Depois $(\overline{\mathrm{X}} \pm \mathrm{DP})$ & $\mathbf{P}$ \\
\hline Coloração da urina & $3,47 \pm 2,17$ & $2,33 \pm 1,40$ & $0,0831^{* *}$ \\
\hline $\mathrm{GEU}$ & $1017,47 \pm 10,01$ & $1009,33 \pm 6,26$ & $<0,01^{* *}$ \\
\hline $\mathrm{Hb}$ & $14,77 \pm 0,88$ & $15,77 \pm 1,23$ & $<0,01^{*}$ \\
\hline $\mathrm{Ht}$ & $43,31 \pm 3,03$ & $46,73 \pm 3,13$ & $<0,01^{*}$ \\
\hline
\end{tabular}

$\overline{\mathrm{X}}$ : média; DP: desvio-padrăo; $p$ : probabilidade de significância; GEU: gravidade específica da urina; $\mathrm{Hb}$ : hemoglobina; Ht: hematócrito; $\left(^{*}\right)$ : teste $t$ de Student pareado; $\left(^{* *}\right)$ : teste de Wilcoxon.

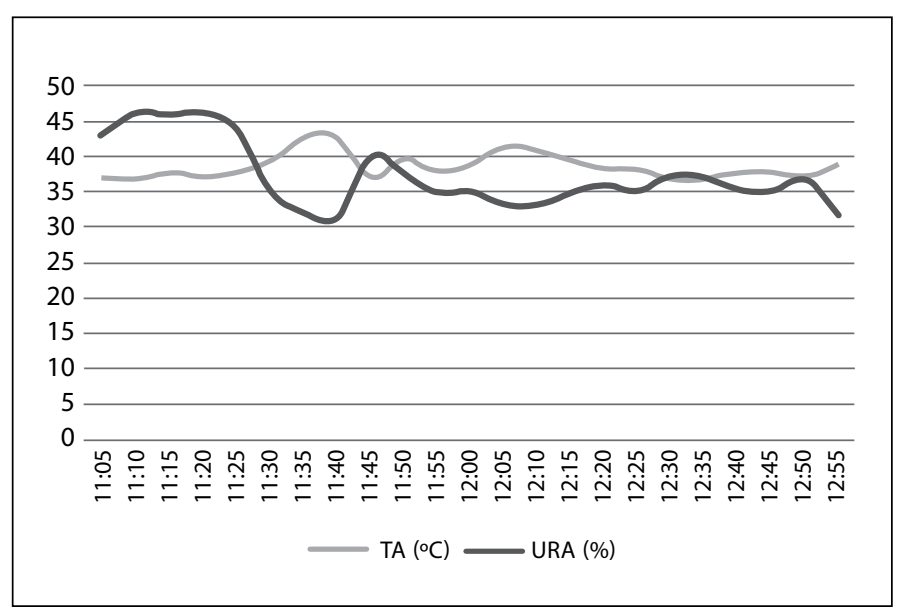

Figura 1. Temperatura ambiente (TA) e Umidade relativa do ar (URA) durante a corrida.

\section{DISCUSSÃO}

Podem-se definir três categorias de testes utilizados para avaliar o estado de hidratação: testes laboratoriais, medidas objetivas não invasivas e observações subjetivas. Todos os métodos têm suas vantagens e desvantagens, existindo testes mais precisos e outros mais práticos para análise ${ }^{9,10}$. Portanto, não existe método infalível para avaliar a hidratação ${ }^{11}$. Muito embora, o estado de hidratação possa ser determinado através de diversos biomarcadores simples que isoladamente tem marcantes limitações, mas que utilizados em conjunto podem fornecer informações valiosas ${ }^{1}$. O presente estudo avaliou o estado de hidratação dos corredores através da variação do peso corporal, coloração da urina, GEU, BIA, níveis de Hb e Ht. Contudo, os resultados apresentados foram conflitantes.

Não existe consenso universal acerca do estado ideal de hidratação antes do exercício, assim como não existe um bom índice de euidratação que possa ser aplicado ${ }^{12}$. A análise da coloração da urina dos indivíduos $(3,47 \pm 2,17)$ foi melhor que o encontrado por Carter et al. ${ }^{13}$ para jovens militares árabes $(5 \pm 2)$ e indica que os indivíduos estavam inicialmente hidratados (coloração da urina $\leq 4$ ) 8,14 . O valor obtido para GEU $(1017,47 \pm 10,01)$ se encontra dentro dos limites estabelecidos na literatura para euidratação ( $\leq 1020^{1,8}$ ou entre 1013 e 102915). A avaliação através da BIA evidenciou um valor de água corporal total $\left(A C T=38,39 \pm 4,73 \mathrm{~L}\right.$ ) inferior ao encontrado por Rossi et al. ${ }^{16}$ e por Sengun et al. ${ }^{17}$ para homens praticantes de mountain bike $(42,0$ $\pm 3,6 \mathrm{~L})$ e mergulhadores profissionais $(46,2 \pm 4,0 \mathrm{~L})$, respectivamente. No entanto, esse valor correspondente a $59,80 \%$ do peso corporal, o que é compatível com indivíduos do sexo masculino e idade entre 19 e 50 anos $(43-73 \%)^{18}$. Os valores de $\mathrm{Ht}(43,31 \pm 3,03)$ encontram-se dentro de valores compatíveis com uma população masculina de região metropolitana, o que também corrobora o estado de adequada hidratação apontado pelos outros métodos ${ }^{19}$.

A diminuição significativa do peso corporal após a prova $(\Delta \%=$ $-3,43 \pm 0,84 \% ; p<0,0001)$ indica que a reposição hídrica ad libitum não foi suficiente para prevenir a desidratação dos indivíduos. De fato, a ingestão de líquidos no decorrer do exercício $(388,61 \mathrm{~mL} / \mathrm{h})$ foi menor que o encontrado, por exemplo, por Godek et al. ${ }^{20}$ para atletas de futebol americano $(1423,80 \mathrm{ml} / \mathrm{h})$ e corredores de cross country $(573 \mathrm{~mL} / \mathrm{h})$. O Colégio Americano de Medicina Esportiva orienta que programas individualizados de reposição fluida devam ser desenvolvidos de forma que as reduções do peso corporal de base sejam menores que $2 \%{ }^{1}$. A perda de massa corporal é a única medida indireta realista de hipo-hidratação para o atleta e o profissional de campo ${ }^{21}$. Evidências atuais sugerem que uma perda de 
peso corporal maior que 2\%, induzida pelo exercício em condições reais de corrida, não comprometa adicionalmente o desempenho de endurance em relação à manutenção da perda de peso corporal em níveis menores ou iguais a $2 \%^{22}$. Contudo, a perda de peso entre $3 \%$ e $6 \%$ é capaz de promover prejuízo a termorregulação, aparecimento de câimbras, contraturas e colapso ${ }^{2}$. O'Neal et al. ${ }^{23}$, sugerem que os participantes de meias-maratonas e maratonas devam ser encorajados a mensurar as modificações de peso corporal durante o exercício visando desenvolver planos individuais de hidratação, uma vez que apenas $11 \%$ dos atletas avaliados treinavam com a supervisão de um técnico ou staff médico. Ademais, 45\% dos participantes do inquérito relataram já ter experimentado sintomas de distúrbios relacionados ao calor, os quais eles pensaram terem sido causados por desidratação.

A melhora do padrão de coloração da urina após a prova não foi estatisticamente significativa ( $p=0,0831)$. Todavia, houve diminuição significativa da GEU após a corrida $(p<0,01)$. Tal situação contrapõe-se ao observado por Ferreira et al. ${ }^{24}$, que observaram elevação da GEU em atletas após o exercício. No entanto, os autores alertam para a necessidade de se avaliar sempre em conjunto a GEU e a variação do peso corporal, em razão da menor exatidão das medidas de GEU em relação a outros métodos e interferência da ingestão de grandes quantidades de líquido antes da avaliação. O consumo de líquidos pode produzir temporariamente uma amostra de urina que não reflita o atual estado de hidratação, pelo fato de os rins poderem filtrar o líquido consumido pouco tempo antes do teste ${ }^{25}$.

As análises urinárias podem ocasionar uma interpretação equivocada do estado de hidratação, devido sua baixa especificidade e relação com a massa muscular dos indivíduos ou ainda com diferenças culturais ${ }^{26,27}$. Por exemplo, a GEU e a osmolalidade urinária respondem a modificações agudas no estado de hidratação, todavia as mudanças nesses marcadores podem ser atrasadas ou insensíveis para baixos níveis de desidratação ( $1 \%$ e $3 \%$ do peso corporal) ${ }^{14}$. Sendo assim, os parâmetros urinários podem fornecer informações enganosas quando obtidos durante períodos de reidratação, como amostras de urina de coloração clara, valores de GEU e osmolalidade urinária que refletem euidratação, quando na verdade o indivíduo permanece desidratado. Portanto, para permitir a adequada discriminação entre os estados de euidratação e desidratação, é recomendada a utilização da primeira amostra de urina da manhã ou ainda de amostras após várias horas de estabilidade do estado de hidratação'. Muito embora, Perrier et al. ${ }^{28}$ tenham demonstrado recentemente que os biomarcadores da primeira urina da manhã não estejam fortemente correlacionados ao consumo fluido.

A avaliação da composição corporal pela BIA não permite afirmar que a diminuição do peso corporal observada deva-se a modificações no conteúdo de água corporal dos indivíduos. Não houve diferença significativa entre o conteúdo de ACT ( $p=0,8647)$ ou mesmo entre os compartimentos intracelular $(p=0,2014)$ e extracelular ( $p=0,0468$ ), na comparação entre os momentos pré e pós-prova. Situação semelhante foi observada por Passaglia et al. ${ }^{29}$ ao avaliar corredores do sexo masculino antes e após uma ultramaratona de 24 horas, tendo sido considerada a possibilidade de redução da acurácia da bioimpedância na medida da ACT imediatamente após exercício intenso.

Muito embora, o padrão ouro para determinação do estado de hidratação seja a medição da ACT, estudos demonstram que BIA e espectroscopia de impedância bioelétrica falham em predizer acuradamente reduções na ACT após rápida desidratação. Os dados aqui obtidos através de BIA poderiam conduzir a conclusão equivocada de que a redução no peso corporal dos indivíduos foi decorrente a uma diminuição do conteúdo total de gordura corporal, uma vez que houve redução da massa gorda após o exercício $(p<0,01)$, quando na verdade sabemos que a perda de peso imediata após o exercício resulta de desidratação ${ }^{14}$. Estudos de validação estabeleceram o método de frequência única como confiável e válido, contudo diversos fatores ambientais e relacionados ao indivíduo podem reduzir a confiabilidade e acurácia da técnica. Tais fatores incluem o local de colocação dos eletrodos, temperatura da pele, fluxo sanguíneo cutâneo, postura, ingestão recente de fluidos, exercício, e mudanças na osmolaridade plasmática ou na concentração plasmática de sódio ${ }^{11}$. Fatores não controlados pelo presente estudo, como aumento da temperatura central ou fluxo sanguíneo cutâneo, podem ter prejudicado a acurácia do método por influenciar as medidas de reatância e resistência nas quais se baseiam tais técnicas.

A elevação nos níveis de Hb e Ht após o exercício $(p<0,01)$ corrobora o estado de desidratação apontado pela variação do peso corporal. A análise da Hb reside sobre os eritrócitos e não deixa a circulação, portanto modificações na sua concentração representam mudanças no volume plasmático ${ }^{11}$. Dessa forma, o aumento dos níveis de Hb após a prova deve refletir diminuição do volume plasmático intravascular. O mesmo raciocínio pode ser estendido à elevação do Ht após o exercício, uma vez que os níveis de Hb e Ht estão tipicamente elevados quando o volume plasmático está reduzido em decorrência à desidratação, podendo ser utilizados na avaliação das mudanças relativas no volume plasmático baseadas na perda de fluidos do espaço vascular. Entretanto, esta técnica tem várias limitações e nem sempre reflete mudanças na hidratação ${ }^{14}$. O aumento significativo dos níveis de $\mathrm{Ht}(p<0,01)$ foi similar ao observado por Ferreira et al. ${ }^{24}$ para indivíduos ativos, após corrida de 80 minutos em esteira ergométrica, o que reforça o fato dos indivíduos serem apenas praticantes habituais de corrida de rua, uma vez que o referido estudo não observou elevação significativa no $\mathrm{Ht}$ de atletas.

O ambiente termoneutro ( $T A=21-24^{\circ} \mathrm{C}$ e URA=50-75\%) é aquele no qual a taxa metabólica de repouso está no seu limite inferior e os mecanismos termorregulatórios não estão sendo demasiadamente exigidos, sendo assim mais adequado à prática de exercícios físicos ${ }^{30}$. Contudo, as condições ambientais registradas não permitiram classificar o ambiente como termoneutro, ganhando importância assim o fato de 10 indivíduos (40\%) terem abandonado a corrida antes de concluir os $15 \mathrm{~km}$ propostos, haja vista o elevado estresse ambiental tenha sido um dos principais fatores implicados pelos indivíduos na desistência.

As recomendações quanto ao cancelamento de qualquer atividade atlética baseiam-se na utilização do índice de bulbo úmido e temperatura de globo (IBUTG) obtido através de um psicrômetro ${ }^{5}$. Portanto, a utilização do referido equipamento talvez fosse mais adequada na monitoração do estresse ambiental. Da mesma forma, um aparelho de bioimpedância por espectroscopia poderia fornecer também maior acurácia na determinação dos compartimentos fluidos corporais. Tais aspectos podem ser entendidos como limitações técnicas do estudo. A determinação da linha de base do peso corporal que representa euidratação pode ser importante para futuros estudos, dada a sua fácil obtenção através de três medidas consecutivas do peso corporal nu pela manhã logo após urinar ${ }^{1}$. Dessa feita, seria possível indicar se os indivíduos já iniciaram a prova em déficit de peso corporal. Em nova oportunidade, a ingestão hídrica deve ser mais bem controlada, uma vez que a ingestão de grandes quantidades de líquido antes do encerramento da coleta de dados pode ter interferido sobre a análise de algumas variáveis. 


\section{CONCLUSÃO}

Os dados aqui apresentados deixam evidentes as dificuldades em relação à monitoração do estado de hidratação em condições reais de prática esportiva. $\mathrm{O}$ estado de desidratação dos indivíduos somente foi detectado através da análise da variação do peso corporal e dos níveis de $\mathrm{Hb}$ e Ht. As técnicas de análise da coloração da urina, GEU e BIA, provavelmente foram confundidas por variáveis não controladas pelo estudo.
O elevado índice de abandono da prova (40\%) pode ser encarado como evidência subjetiva de desidratação. Sendo assim, parece seguro concluir que a análise conjunta por diferentes técnicas seja a estratégia mais eficiente na determinação do estado de hidratação em corredores de rua.

Todos os autores declararam não haver qualquer potencial conflito de interesses referente a este artigo.

\section{REFERÊNCIAS}

1. American College of Sports Medicine, Sawka MN, Burke LM, Eichner ER, Maughan RJ, Montain SJ, et al. American College of Sports Medicine position stand. Exercise and fluid replacement. Med Sci Sports Exerc. 2007;39(2):377-90

2. Carvalho T, Mara LS. Hidratação e nutrição no esporte. Rev Bras Med Esporte. 2010;16(2):144-8.

3. Wilmore JH, Costill DL. Fisiologia do esporte e do exercício. São Paulo: Manole; 2001.

4. American College of Sports Medicine, Armstrong LE, Casa DJ, Millard-Stafford M, Moran DS, Pyne SW, et al. American College of Sports Medicine position stand. Exertional heat illness during training and competition. Med Sci Sports Exerc. 2007;39(3):556-72.

5. Hernandez AJ, Nahas RM, Rodrigues T, Meyer F, Zogaib P, Lazzoli JK, et al. Modificações dietéticas, reposição hídrica, suplementos alimentares e drogas: comprovação de ação ergogênica e potenciais riscos para a saúde. Rev Bras Med Esporte. 2009;15(3):1-12.

6. Machado-Moreira CA, Vimieiro-Gomes AC, Silami-Garcia E, Rodrigues LOC. Hidratação durante o exercício: a sede é suficiente? Rev Bras Med Esporte. 2006;12(6):405-9.

7. Sociedade Brasileira de Cardiologia, Sociedade Brasileira de Hipertensão, Sociedade Brasileira de Nefrologia. VI Brazilian Guidelines on hypertension. Arq Bras Cardiol. 2010;95(Suppl 1):1-51.

8. Casa DJ, Armstrong LE, Hillman SK, Montain SJ, Reiff RV, Rich BS, et al. National athletic trainers' association position statement: fluid replacement for athletes. J Athl Train. 2000;35(2):212-24.

9. Shirreffs SM. Markers of hydration status. Eur J Clin Nutr. 2003;57(Suppl 2):6-9.

10. Pereira ER, Mendes TT, Pacheco DAS, Alves AL, Melo MAA, Silami-Garcia E. Hidratação: conceitos e formas de avaliação. e-Scientica. 2010;3(2):13-24

11. Armstrong LE. Hydration assessment techniques. Nutr Rev. 2005;63(6 Pt 2):S40-54.

12. Maughan RJ, Shirreffs SM. Dehydration and rehydration in competative sport. Scand J Med Sci Sports. 2010;20(Suppl 3):40-7.

13. Carter JM, Loney T, Blacker SD, Nicholson GF, Wilkinson DM. Hydration status of Arabic adolescents and young men: measurement, evaluation, and a school-based initiative to improve drinking behavior. Int J Sport Nutr Exerc Metab. 2012;22(4):257-66.

14. Turocy PS, DePalma BF, Horswill CA, Laquale KM, Martin TJ, Perry AC, et al. National Athletic Trainers' Association position statement: safe weight loss and maintenance practices in sport and exercise. J Athl Train. 2011;46(3):322-36.

15. Armstrong LE, Maresh CM, Castellani JW, Bergeron MF, Kenefick RW, LaGasse KE, et al. Urinary indices of hydration status. Int J Sport Nutr. 1994;4(3):265-79.
16. Rossi L, Candelária VKC, Gomes PS.Perfil antropométrico e nutricional de praticantes de moutain bike. Braz J Biomotricity. 2010;4:180-9.

17. Sengun S, Uslu A, Aydin S. Application of multifrequency bioelectrical impedance analysis method for the detection of dehydration status in professional divers. Medicina (Kaunas). 2012;48(4):203-10.

18. Noujeimi FA. Avaliação da água corporal total e seus compartimentos em atletas de elite por espectrometria de impedância [dissertação]. Porto: Universidade do Porto; 2012.

19. Azevedo AP, Silva P, Marcelo C, Gamelas C, Teixeira V, Vieira A, et al. Hemogram reference values for Lisbon metropolitan área population. Acta Med Port. 2010;23(4):597-604

20. Godek SF, Godek JJ, Bartolozzi AR. Hydration status in college football players during consecutive days of twice-a-day preseason practices. Am J Sports Med. 2005;33(6):843-51.

21. Maughan RJ, Shirreffs SM, Leiper JB. Errors in the estimation of hydration status from changes in body mass. J Sports Sci. 2007;25(7):797-804.

22. Goulet ED. Dehydration and endurance performance in competitive athletes. Nutr Rev. 2012;70(Suppl 2):132-6.

23. O'Neal EK, Wingo JE, Richardson MT, Leeper JD, Neggers YH, Bishop PA. Half-marathon and full-marathon runners' hydration practices and perceptions. J Athl Train. 2011;46(6):581-91.

24. Ferreira FG, Alves K, Costa NMB, Santana AMC, Marins JCB. Efeito do nível de condicionamento físico e da hidratação oral sobre a homeostase hídrica em exercício aeróbico. Rev Bras Med Esporte. 2010;16(3):166-70.

25. Popowski LA, Oppliger RA, Patrick Lambert G, Johnson RF, Kim Johnson A, Gisolf CV. Blood and urinary measures of hydration status during progressive acute dehydration. Med Sci Sports Exerc. 2001;33(5):747-53.

26. Manz F, Wentz A. 24-h hydration status: parameters, epidemiology and recommendations. Eur J Clin Nutr. 2003;57(Suppl 2):S10-8.

27. Hamouti N, Del Coso J, Avila A, Mora-Rodriguez R. Effects of athletes' muscle mass on urinary markers of hydration status. Eur J Appl Physiol. 2010;109(2):213-9.

28. Perrier E, Rondeau P, Poupin M, Le Bellego L, Armstrong LE, Lang F, et al. Relation between urinary hydration biomarkers and total fluid intake in healthy adults. Eur J Clin Nutr. 2013;67(9):939-43.

29. Passaglia DG, Emed LG, Barberato SH, Guerios ST, Moser Al, Silva MM, et al. Acute effects of prolonged physical exercise: evaluation after a twenty-four-hour ultramarathon. Arq Bras Cardiol. 2013;100(1):21-8.

30. Garcia ES, Rodrigues LOC. Hipertermia durante a prática de exercícios físicos: riscos, sintomas e tratamento. Rev Bras Cienc Esporte. 1998;19(3):85-94. 